THE aim of this study was to determine phenotypic differences when BCG invades macrophages. Bacilli prepared from the same BCG primary seed, but produced in different culture media, were analysed with respect to the ability to stimulate macrophages and the susceptibility to treatment with cytokines and nitric oxide (NO). Tumour necrosis factor (TNF) activity was assayed by measuring its cytotoxic activity on L-929 cells, in terleukin-6 (IL-6) and in terferon$\gamma($ IFN- $\gamma)$ were assayed by enzyme-linked immunosorbent assay (ELISA), whereas NO levels were detected by Griess colorimetric reactions in the culture supernatant of macrophages incubated with IFN- $\gamma$, TNF or NO and subsequently exposed to eith er BCG-I or BCG-S. We found th at BCG-I and BCG$S$ bacilli showed different ability to simulate peritoneal macrophages. Similar levels of IL-6 were detected in stimulated macrophages with lysate from two BCG samples. The highest levels of TNF and IFN- $\gamma$ were observed in macrophages treated with BCG-S and BCG-I, respectively. The highest levels of NO were observed in cultures stimulated for $48 \mathrm{~h}$ with BCG-S. We also found a different susceptibility of the bacilli to exogenous treatment with IFN- $\gamma$ and TNF which were capable of killing 60 and $70 \%$ of both bacilli, whereas NO was capable of killing about 98 and $47 \%$ of BCG-I and BCG-S, respectively. The amount of bacilli proportionally decreased with IFN$\gamma$ and TNF, suggesting a cytokine-related cytotoxic effect. Moreover, NO also decreased the viable number of bacilli. Interestingly, NO levels of peritoneal macrophages were sign ificantly increased after cytokine treatment. This indicates that the treatment of macrophages with cytokines markedly reduced bacilli number and presented effects on NO production. The results obtained here emphasize the im portance of adequate stimulation for guaranteeing efficient killing of bacilli. In this particular case, the IFN- $\gamma$ and TNF were involved in the activation of macrophage bactericidal activity.

Key words: Cytokines, Nitric oxide, BCG vaccines, Macrophage

\section{Role of cytokines and nitric oxide in the induction of tuberculostatic macrophage functions}

\author{
Vera L. Petricevich ${ }^{1, C A}$ and Rosely C. B. Alves ${ }^{2}$ \\ 'Laboratorio de Imunoquímica, Instituto Butantan; \\ ${ }^{2}$ Laboratório de Produção de BCG, Instituto \\ Butantan, São Paulo, Brazil \\ ${ }^{\mathrm{CA}}$ Corresponding Author \\ Email: velupetri@hotmail.com
}

\section{Introduction}

Mycobacteria are intracellular pathogens which preferentially reside in resident macrophages, whereas activated macrophages are presumed to eliminate the bacteria effectively. Mycobacterium tuberculosis, the causative agent of tuberculosis, enters the macrophage via binding to several distinct cell surface molecules. Following phagocytosis, sustained intracellular bacterial growth depends on the ability to avoid destruction by macrophage-mediated host defences such as lysosomal enzymes, reactive oxygen, reactive nitrogen intermediates and cytokines. Resistance to tuberculosis crucially depends on specific $\mathrm{T}$ cells which activate intracellular killing of the infectious agent by macrophages. ${ }^{1} \mathrm{CD} 4 \mathrm{~T}$ cells comprise two functionally distinct helper subsets according to their cytokine profiles, namely, Th1 and Th2 cells. ${ }^{2-4}$ Predominance of Th1 or Th2 cell responses has an important influence on the outcome of infection with intracellular pathogens. ${ }^{5}$ The development of the Th cell type is influenced by several cytokines, such as interleukin- 4 (IL-4) and interferon-g $(\mathrm{IFN}-\mathrm{g}),{ }^{2}$ which are produced at the onset of an immune response and are thought to be decisive for pathological or physiological consequences. ${ }^{6}$ The release of cytokines by macrophages has shown that 
excessive or insufficient production may significantly contribute to the pathophysiology of a range of diseases. ${ }^{2,6,7}$ Previous studies have shown that the control of mycobacterial infections depends on the cytokine-mediated activation of mononuclear phagocytes to inhibit the growth of intracellular mycobacteria. Recent studies demonstrated that tumour necrosis factor-a (TNF-a) acts as an endogenous cofactor in the induction of mycobacterial growth inhibition. ${ }^{8}$ Murine peritoneal macrophages activated with IFN-g produce large quantities of nitric oxide (NO) and are efficient in killing certain intracellular pathogens. ${ }^{9}$ This study shows the susceptibility and/ or resistance of two kinds of BCG bacilli which were cultivated in two distinct medium: Instituto Viscondessa de Moraes medium (IVM) and Sauton medium, here referred to as BCG-I and BCG-S, respectively. The resistance may reflect in part the ability of these organisms to resist the enhanced bacteriostatic and bactericidal properties acquired by host macrophages as a result of these mycobacterial infections.

\section{Materials and methods}

\section{Chemicals, reagents and buffers}

RPMI-1640 medium, actinomycin D, orthophenyldiamine (OPD), sodium nitrate (NO) were purchased from Sigma (St. Louis, MO, USA), fetal calf serum (FCS) was purchased from Cutilab, Campinas,SPBrazil, murine anti-IL-6 (clones: MP5-20F3 and MP532.C.11), rIL-6, anti-IFN-g (clones XGM1.2 and AN18), rIFN-g were purchased from PharMingen (Toreyana, San Diego, USA) and rTNF, $p$-nitrophenylphosphate (pNPP) were purchased from Boehringer Mannheim (Germany).

\section{BCG bacilli}

The BCG used in this study was Moreau, Copenhagen strain, which was stored in a freeze-dried state at $-20^{\circ} \mathrm{C}$ and called BCG primary lots. The secondary seed lots were derived from the primary seed lots and cultivated by two passages on Sauton potato medium before being transferred to the liquid media. They were prepared in batches of samples, using IVM medium for the expansion of BCG-I, while BCG-S was expanded in liquid Sauton medium. ${ }^{10}$ The veils grown on the surface of liquid IVM and Sauton medium for 11 and 7 days, respectively, were used. The bacillary mass was separated from the culture medium, then resuspended in Sauton medium and homogenized by ball-milling. The bacillary mass obtained for the BCG-S preparation was separated from the culture medium, then homogenized by ball-milling and resuspended in sodium glutamate as a protective excipient for freezedrying. The optical density was determined at $390 \mathrm{~nm}$ and $400 \mathrm{~nm}$ for BCG-I and BCG-S, respectively.

\section{Lysates of BCG bacilli}

Lysates of BCG were obtained from bacilli BCG-I and BCG-S. ${ }^{11}$ In brief: $10^{7}$ colony-forming units (cfu) were incubated in $1 \mathrm{ml}$ of saline solution at $60^{\circ} \mathrm{C}$ for $60 \mathrm{~min}$ and sonication was performed for $15 \mathrm{~min}$ at $100 \mathrm{~W}$ to promote cell lysis. The cell lysate thus obtained was stored at $-20^{\circ} \mathrm{C}$ until use. The mixtures obtained were used for macrophage stimulation.

\section{Stimulation of mouse peritoneal macrophages}

BALB/c mice (20-22 g) were obtained from Biotério (Instituto Butantan, SP, Brazil). All animals were maintained under strict ethical conditions according to international recommendations. ${ }^{12}$ Groups of mice were sacrificed and their cells were harvested by peritoneal lavage. ${ }^{13}$ The cells were seeded in 24-well microtitre plates at a concentration of $1 \times 10^{6}$ cells $/ \mathrm{ml}$ and cultured in RPMI-1640 medium supplemented with $10 \%$ FCS. After incubation at $37^{\circ} \mathrm{C}$ for $2 \mathrm{~h}$ in humidified $5 \% \mathrm{CO}_{2}$, the plates were then washed twice with RPMI-1640 medium to remove nonadherent cells and the adherent cells were referred to as macrophages. These cells were exposed to different concentrations of lysate of BCG-I or BCG-S in RPMI1640 containing $10 \% \mathrm{FCS}$. After incubation at $37^{\circ} \mathrm{C}$ for various intervals of time in a humidified atmosphere of $5 \% \mathrm{CO}_{2}$, the supernatants were collected and stored at $-20^{\circ} \mathrm{C}$ until assayed for the presence of cytokines, NO and acid phosphatase activity.

\section{Cytokine determination}

The levels of cytokines IL-6 and IFN-g in the culture supernatants were assayed by two-site sandwich enzyme-linked immunosorbent assay (ELISA). ${ }^{14}$ Briefly, ELISA plates were coated with $100 \mathrm{ml}$ $(1 \mathrm{mg} / \mathrm{ml})$ of the monoclonal antibodies anti-IL-6 or anti-IFNg in $0.1 \mathrm{M}$ sodium carbonate buffer, $\mathrm{pH} 8.2$, and incubated for $6 \mathrm{~h}$ at room temperature. The wells were then washed with $0.1 \%$ phosphate-buffered saline (PBS)/Tween 20 and blocked with $100 \mathrm{ml}$ of FCS in 10\% PBS for $2 \mathrm{~h}$ at room temperature. After washing, duplicate supernatant macrophage culture samples of $50 \mathrm{ml}$ were added to each well. After $18 \mathrm{~h}$ of incubation at $4^{\circ} \mathrm{C}$ the wells were washed and incubated with $100 \mathrm{ml}(2 \mathrm{mg} / \mathrm{ml})$ of the biotinylated monoclonal antibodies anti-IL-6 or anti-IFN-g as second antibodies for $45 \mathrm{~min}$ at room temperature. After a final wash, the reaction was developed by the addition of OPD to each well. Optical densities were measured at $405 \mathrm{~nm}$ in a microplate reader. The cytokine content of each sample was read from a 
standard curve established with the appropriate recombinant cytokine and expressed in $\mathrm{ng} / \mathrm{ml}$. The minimum levels of each cytokine detectable in the conditions of the assays were 0.78 and $3.9 \mathrm{ng} / \mathrm{ml}$ for IL-6 and IFN-g, respectively.

To measure the cytotoxicity of TNF present in the supernatants from the macrophages, a standard assay with L-929 cells, a fibroblast continuous cell line, was used as described previously. ${ }^{15}$ The percentage cytoxicity was calculated as follows: ( $A_{\text {control }}-A_{\text {sample }}$ $\left.A_{\text {control }}\right) \times 100$ and the titres were calculated as the reciprocal of the dilution of the sample in which 50\% of the cells in the monolayers were lysed. TNF activity was expressed in $\mathrm{ng} / \mathrm{ml}$ estimated from the ratio between a $50 \%$ cytotoxic dose of the test and that of standard recombinant mouse TNF.

\section{NO determination}

The levels of NO in supernatants from macrophages treated with BCG-I or BCG-S bacilli were assayed by adding $50 \mathrm{ml}$ of freshly prepared Griess reagent ${ }^{16}$ to $50 \mathrm{ml}$ of the sample in 96-well plates and reading the absorbance at $540 \mathrm{~nm} 10 \mathrm{~min}$ later by comparison with the absorbance curves of serial dilutions of sodium nitrate in complete culture medium. The minimum level of NO detectable under the assay conditions was $1 \mathrm{nmol}$.

\section{Macrophage activation (acid phosphatase activity)}

The acid phosphatase activity of peritoneal macrophages from mice was determined as described previously. ${ }^{17}$ In brief: macrophages obtained and stimulated as described above were placed in a test tube and allowed to stand at $34^{\circ} \mathrm{C}$ for $20 \mathrm{~min}$ to permit macrophage adherence to the glass surface. The tubes were washed three times with PBS, $2 \mathrm{ml}$ of water was added, and sonication was performed for $30 \mathrm{~s}$ at $100 \mathrm{~W}$ to promote cell lysis. To the cell lysate thus obtained $1.5 \mathrm{ml} 0.3 \mathrm{M}$ citrate buffer, $\mathrm{pH} 4.9$, and $0.2 \mathrm{ml} 0.04 \mathrm{M}$ pNPP substrate were added. The mixture was incubated at $37^{\circ} \mathrm{C}$ for $1 \mathrm{~h}$, and $0.5 \mathrm{ml} 1 \mathrm{MTris}$, pH 8.5, containing $0.4 \mathrm{M} \mathrm{K}_{2} \mathrm{PO}_{4}$ was added. The absorbance was then read at $420 \mathrm{~nm}$ using a Pharmacia spectrophotometer. Protein determination in the cell lysate was carried out using the method of Lowry et al. ${ }^{18}$ Enzyme activity was defined as the units of optical density of nitrophenol released per milligram of protein per hour.

\section{cfu assay}

Bacterial loads in the peritoneal macrophages treated with BCG bacilli were evaluated by plating 10-fold serial dilutions of macrophage homogenates in PBS on to Löwenstein-Jensen tubes and incubating at $37^{\circ} \mathrm{C}$ for 28 days. The bacterial colonies of each culture (six replicate samples) were counted and the mean calculated.

\section{Statistical analyses}

Data are expressed as the mean \pm standard deviations (SD). Statistical analyses were performed by Student's $t$-test and the level of significance was set at $p<0.05$.

\section{Results}

\section{Effect of BCG bacilli on macrophage activation}

To compare macrophage activation, mice were sacrificed and the macrophages which had been stimulated with different amounts of viable and lysate BCG were collected by peritoneal lavage. The effects of lysate BCG bacilli upon macrophage stimulation were determined by measuring acid phosphatase activity. Both viable and lysate BCG were capable of increasing macrophage activation. As shown in Fig. 1A, similar levels of activation for all groups of macrophages treated with viable and lysate BCG were observed $24 \mathrm{~h}$ after this treatment. For all groups of mice treated with viable and lysate BCG this level increased with increase in stimulation dose until a plateau was attained.

To analyse the lysate BCG bacilli interference with macrophage activation, cells were obtained from mice and stimulated in vitro with different amounts of lysate BCG. Figure 1B shows that the lysate BCG bacilli showed similar ability to stimulate macrophages in vitro. Macrophage activation was increased with increase in dose of lysate BCG bacilli. With doses of 10 and $50 \mathrm{mg} / \mathrm{ml}$, low stimulation was observed in all groups of macrophages treated with both lysates. In contrast, for groups treated with higher doses, the stimulation started to appear at $100 \mathrm{mg} / \mathrm{ml}$ increasing thereafter until a plateau was attained at $500-1000 \mathrm{mg} / \mathrm{ml}$. Although an increased level of stimulation was observed in all groups, this increase was no different between BCG-I and BCG-S (Fig. 1B).

To determine the kinetics of the effects of lysate BCG bacilli, cells were obtained from mice and stimulated in vitro with $100 \mathrm{mg} / \mathrm{ml}$ of lysate BCG (Fig. 1C). The highest macrophage activation by BCG-I occurred $24 \mathrm{~h}$ post-treatment. In contrast, the highest activation by BCG-S occurred around $48 \mathrm{~h}$ posttreatment. The levels of activation were almost twice as high in macrophages stimulated in vitro with lysate BCG-S when compared with those obtained for macrophages treated with lysate O-BCG (Fig. 1C).

\section{Effect of lysate BCG bacilli in in vitro activation}

To compare cytokine production, groups of mice were sacrificed and their macrophages, which were collected by peritoneal lavage, were stimulated in 


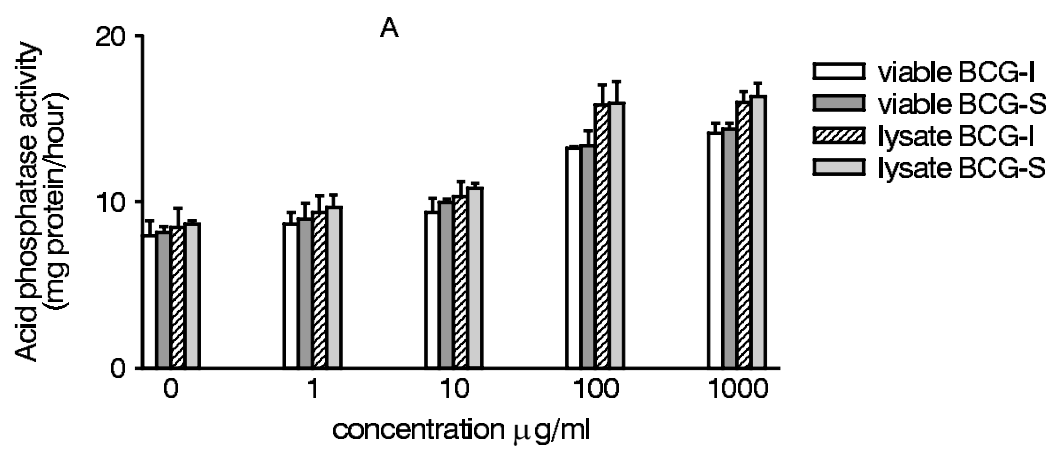

B

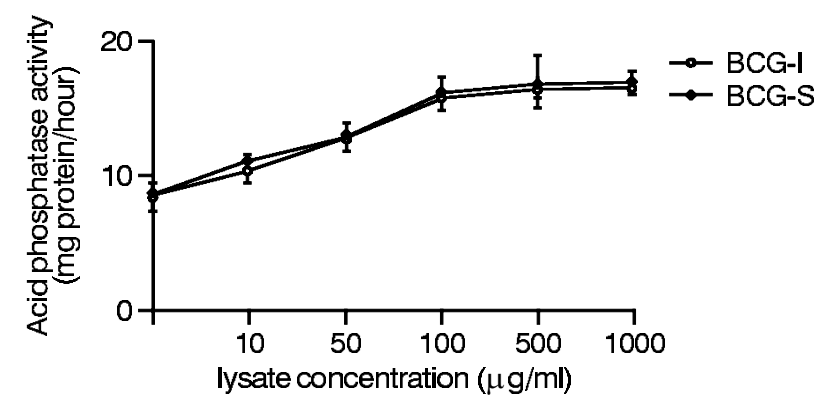

C

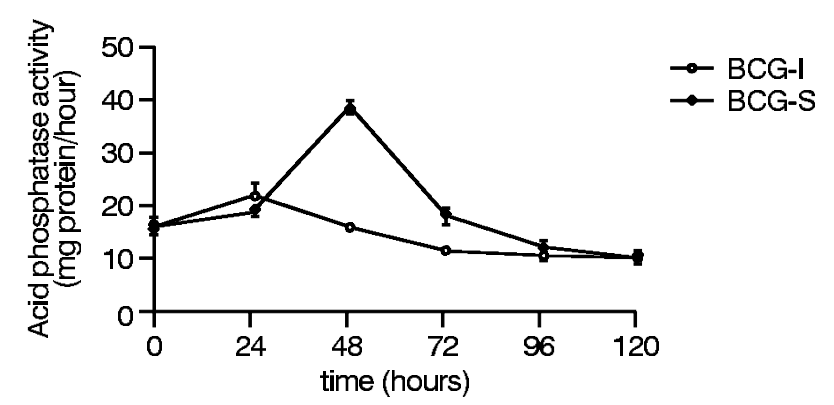

FIG. 1. Macrophage activation. (A) Groups of BALB/c mice were sacrificed and their peritoneal macrophages collected and stimulated in vitro with $100 \mathrm{mg}$ of viable or lysate BCG bacilli for $48 \mathrm{~h}$ and activation was determined by measurement of acid phosphatase activity. (B) Peritoneal macrophages from mice were obtained and stimulated in vitro for $48 \mathrm{~h}$ with different amounts of BCG lists. (C) Peritoneal macrophages from mice were obtained and stimulated in vitro for different times with $100 \mathrm{mg} / \mathrm{ml}$ of BCG lysates. Each point represents the values of samples from five experiments \pm standard deviation in different groups of five mice.

vitro with $100 \mathrm{mg} / \mathrm{ml}$ lysate BCG bacilli (Fig. 2). The levels of IFN-g increased until $72 \mathrm{~h}$ in all macrophage groups stimulated in vitro with both lysates (Fig. 2A). The levels of IFN-g in groups of macrophages stimulated in vitro with lysate BCG-I were higher when compared with those obtained in cultures stimulated with lysate BCG-S $(p>0.001)$. Figure 2B shows that the levels of TNF started to appear after $24 \mathrm{~h}$ in all cultures, decreasing thereafter. The highest levels of TNF were observed in macrophages stimulated in vitro for $48 \mathrm{~h} \mathrm{~h}$ for both lysates. The levels of TNF in groups of macrophages stimulated in vitro with lysate BCG-I were significantly lower when compared with those obtained in cultures stimulated with BCG-S $(p>0.01)$. As shown in Fig. 2C, similar levels were detected in macrophages stimulated in vitro with lysate from both bacilli. The maximum production of IL- 6 was detected in cultures stimulated for $24 \mathrm{~h}$.

To determine NO production, groups of mice were sacrificed and macrophages collected by peritoneal lavage were stimulated in vitro with $100 \mathrm{mg} / \mathrm{ml}$ of lysate BCG bacilli (Fig. 3). The levels of NO increased until 48 and $72 \mathrm{~h}$ for BCG-I and BCG-S, respectively. The levels of NO in macrophages stimulated in vitro with lysate BCG-S were significantly higher than those obtained in cultures stimulated with BCG-I $(p>0.001)$.

\section{Growth inhibition of BCG bacilli in macrophages}

To evaluate the growth of viable BCG bacilli in macrophages, groups of mice were sacrificed and 

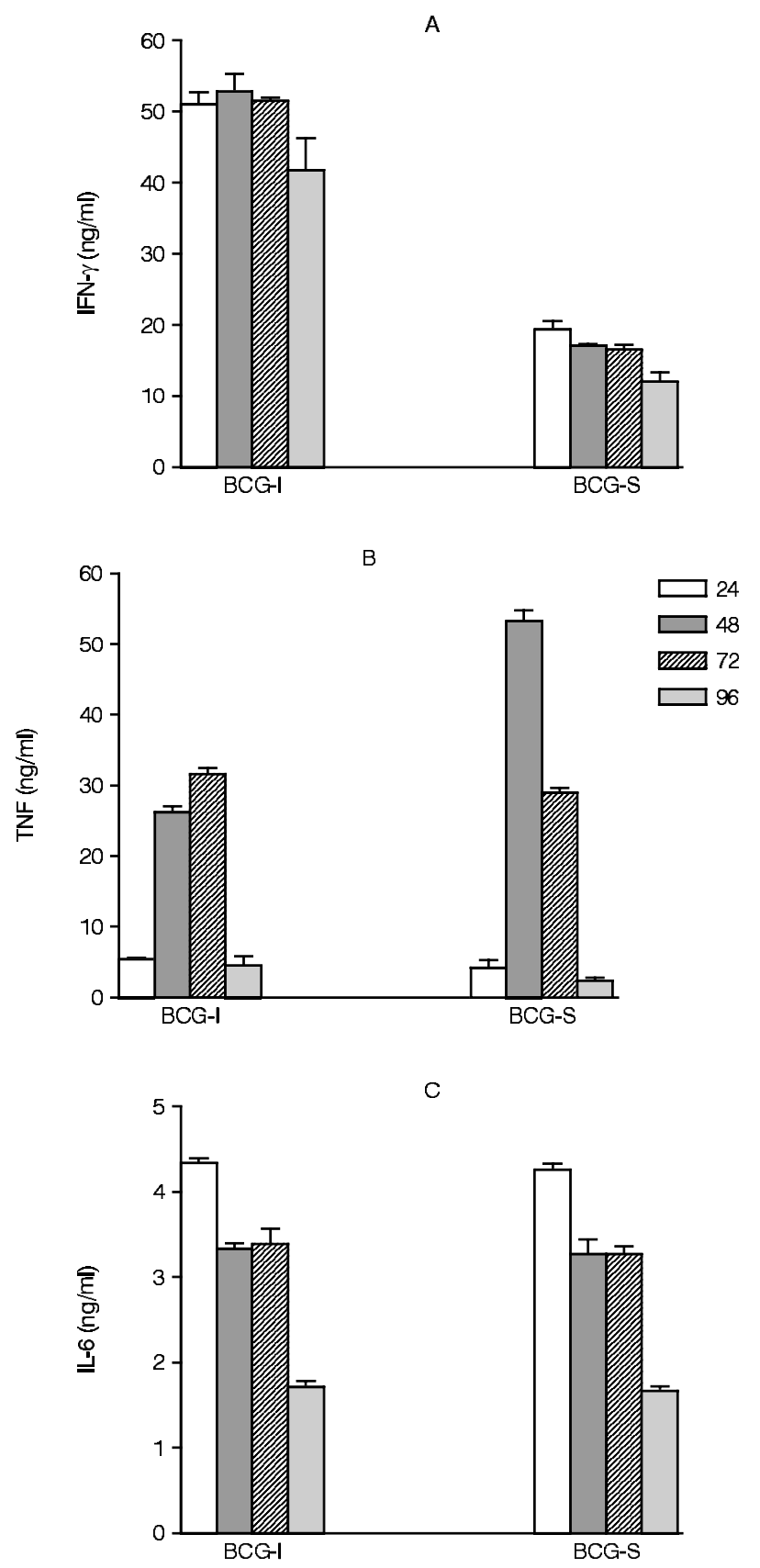

FIG. 2. Cytokine released by peritoneal macrophages from $B A L B / c$ mice. Mice were sacrificed and their peritoneal macrophages were collected. Peritoneal macrophages were stimulated in vitro with $100 \mathrm{mg}$ of BCG lysates and at different time intervals the supernatants were collected. Tumour necrosis factor activity was assayed by measuring its cytotoxic activity on L-929 cells, whereas interleukin-6 and interferon-g were assayed by enzyme-linked immunosorbent assay using monoclonal antibodies as the probe. Each point represents the values of samples from five experiments \pm standard deviation in different groups of five mice.

macrophages collected by peritoneal lavage. The cells were infected in vitro with $1.5 \times 10^{6} \mathrm{cfu}$ of viable BCG bacilli for 24, 48, 72, 96 and $120 \mathrm{~h}$. Afterwards macrophage homogenates were obtained to determine the viable bacilli number. The number of bacilli in macrophages was determined by plating the suspension on Löwenstein-Jensen tubes, and counting

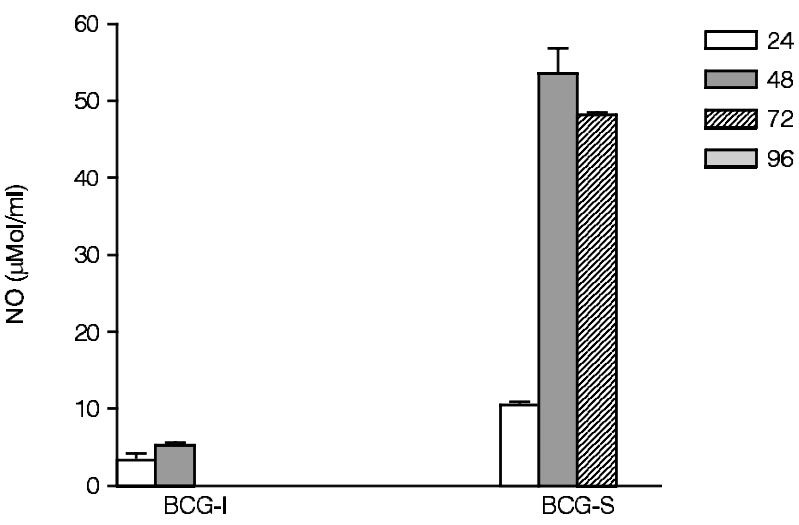

FIG. 3. Nitric oxide (NO) production. Mice were sacrificed and their peritoneal macrophages were collected. Peritoneal macrophages were stimulated in vitro as described above. NO levels were detected by Griess colorimetric reaction. Each point represents the values of samples from five experiments \pm standard deviation in different groups of five mice.

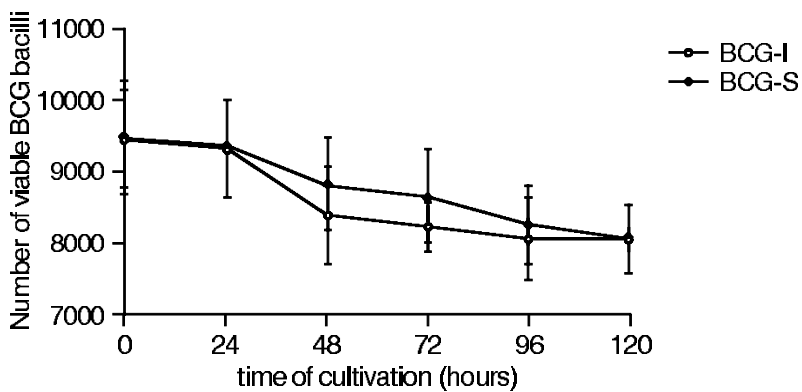

FIG. 4. Growth inhibition of BCG bacilli in peritoneal macrophages. Groups of five mice were sacrificed and their peritoneal macrophages collected and infected with $1.5 \times 106$ colony-forming units of BCG-I or BCG-S. At different times the number of intracellular bacilli was assessed as described in Materials and Methods. Each point represents the values of samples from five experiments \pm standard deviation in different groups of five mice.

the cfu 28 days later. The number of viable BCG-I and BCG-S recovered from macrophages decreased with increasing culture time in all groups (Fig. 4).

\section{Susceptibility of BCG to cytokines}

To determine the susceptibility of BCG to cytokines, macrophages from mice were collected and separated into three groups. In the first group, referred to as 'before', the macrophages were treated with $125 \mathrm{mg}$ of recombinant cytokines for $24 \mathrm{~h}$ and infected with $1.5 \times 10^{6} \mathrm{cfu}$ of BCG-I or BCG-S. In the second group, 'during', the macrophages were treated with the same concentration of recombinant cytokines and infected with same dose of BCG bacilli. In the third group, 'after', the macrophages were infected with $1.5 \times 10^{6}$ cfu of BCG bacilli for $24 \mathrm{~h}$ and then treated with $125 \mathrm{mg}$ of recombinant cytokines. As shown in Table 1 , the lowest viable bacilli number was observed in macrophages from the 'before' group. In contrast, the 
highest number of viable BCG was observed for the groups 'during' and 'after' (Table 1).

After establishment, an optimal time stimulation resulted in the lowest number of viable BCG, this implies that, given a certain amount of recombinant cytokine available for infection, there should exist an optimal concentration and consequently less bacilli. Macrophages were obtained and treated in vitro with different concentrations of recombinant cytokines alone or combined for $24 \mathrm{~h}$, followed by BCG infection. The rate of growth inhibition was assessed by lysing the macrophages $48 \mathrm{~h}$ after this treatment and counting the number of cfu in Löwenstein-Jensen tubes 28 days later. Figure $5 \mathrm{~A}$ shows that at $125 \mathrm{mg}$ of IFN-g the macrophages were capable of killing around
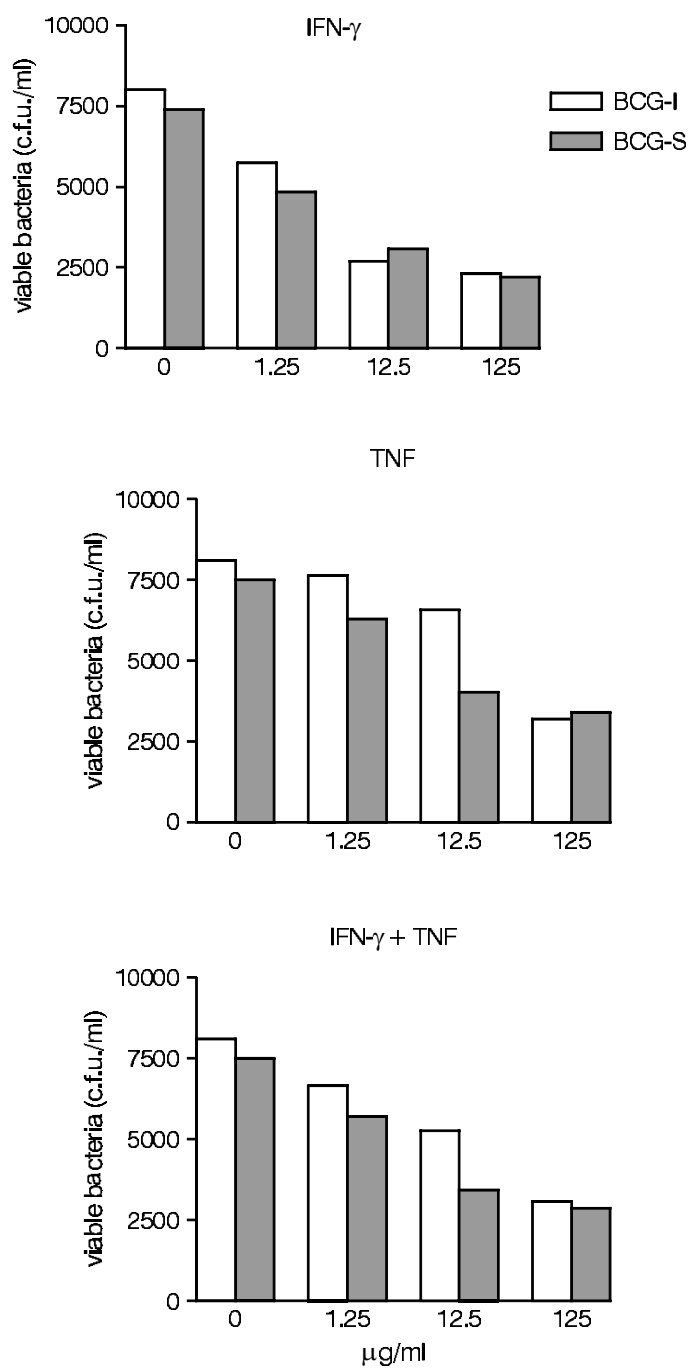

FIG. 5. Growth inhibition and susceptibility of BCG bacilli. Groups of five mice were sacrificed and their peritoneal macrophages were collected. Macrophages were treated with different concentrations of interferon-g (IFN-g), tumour necrosis factor (TNF) or IFN-g + TNF for $24 \mathrm{~h}$ and infected with $1.5 \times 10^{6}$ colony-forming units of viable BCG bacilli. The number of intracellular bacilli was assessed as described in Materials and Methods. Each point represents the values of samples from five experiments \pm standard deviation in different groups of five mice.
Table 1. Bacilli number

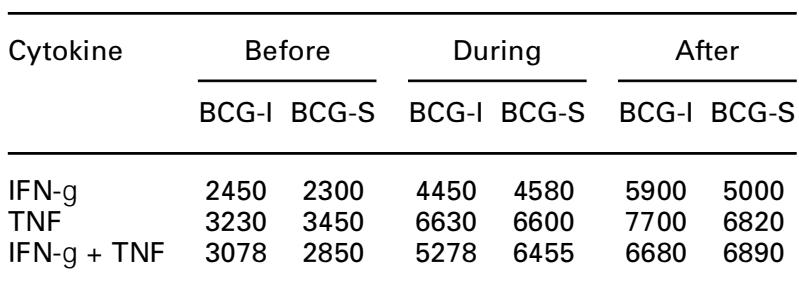

IFN, interferon; TNF, tumour necrosis factor.

$70 \%$ of both bacilli. Figure $5 \mathrm{~B}$ shows that $125 \mathrm{mg}$ of TNF was capable of killing about 60 and 54\% of BCG-I and BCG-S, respectively. Figure 5C shows that TNF plus IFN-g was capable of killing about $62 \%$ of both BCG bacilli.

In order to verify if the exogenous cytokine showed a cytotoxic effect on BCG bacilli, NO levels were determined (Fig. 6). The levels of NO increased with increasing cytokine dose for all groups. With a dose of $125 \mathrm{mg} / \mathrm{ml}$ of IFN-g, TNF alone or combined, the levels of NO were highest when compared with the levels obtained in untreated macrophages.

\section{Susceptibility of BCG to NO}

To determine if NO plays a role in the susceptibility of bacilli in the absence of the other products of activated effector cells, the level of mortality of bacilli in the presence of NO was examined. $1.5 \times 10^{6} \mathrm{cfu}$ of BCG-I or BCG-S were exposed at different concentrations of NO for $24 \mathrm{~h}$. The lowest number of viable bacilli were observed when macrophages were treated with NO (Fig. 7). Doses of 1.9 and $3.75 \mathrm{mmol}$ of NO were capable of killing around $20 \%$ of both bacilli. In contrast, when macrophages were treated with $7.5 \mathrm{mmol}$ of $\mathrm{NO}$, the number of killed bacilli was 47 and $43 \%$ for BCG-I and BCG-S, respectively. The number of killed BCG-S bacilli observed in macrophage groups treated with $15 \mathrm{mmol}$ of $\mathrm{NO}$ was around

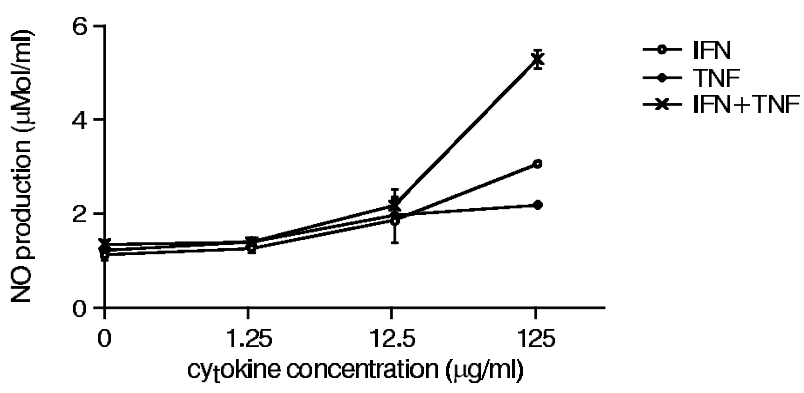

FIG. 6. Cytotoxic effect of cytokines. Groups of five mice were sacrificed and their peritoneal macrophages were collected. Macrophages were treated with different concentrations of interferon-g (IFN-g), tumour necrosis factor (TNF) or IFN-g + TNF for $24 \mathrm{~h}$ and infected with $1.5 \times 10^{6}$ colony-forming units of viable BCG bacilli. Nitric oxide (NO) levels were assayed by the Griess colorimetric reactions. Each point represents the values of samples from five experiments \pm standard deviation in different groups of five mice. 


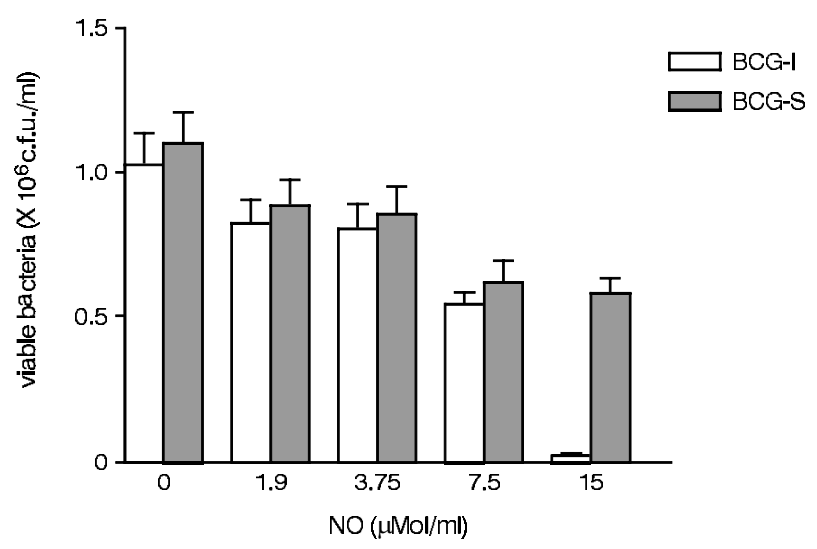

FIG. 7. Susceptibility of BCG bacilli to nitric oxide (NO): $1.5 \times$ $10^{6}$ colony-forming units of BCG-I or BCG-S were exposed to different amounts of NO for $24 \mathrm{~h}$. The number of viable BCG-I or BCG-S was assessed as described in Materials and Methods. Each point represents the values of samples from five experiments \pm standard deviation in different groups of five mice.

$47 \%$, while the number of killed BCG-I bacilli observed in macrophages treated with $15 \mathrm{mmol}$ of $\mathrm{NO}$ was $98 \%$ (Fig. 7).

\section{Discussion}

Historically, researchers have assumed that activated macrophages can kill M. tuberculosis. However, this assumption has been difficult to prove unequivocally in vitro, especially with human monocytes and macrophages. Human monocytes cultured for 3 days were measurably better at suppressing the growth of virulent $M$. tuberculosis than were either fresh monocytes or those cultured for 7 days. ${ }^{19}$ Following treatment with cytokines such as IFN-g andTNF, human monocytes can be activated showing an intense microbicidal. ${ }^{20}$ However, a more recent report suggested that this apparent killing of $M$. tuberculosis could be an artefact of the experiment and that this cytokine treatment regimen actually renders macrophages more sensitive to the toxic effects of the mycobacteria. $^{21}$

Mycobacteria are intracellular pathogens which survive and grow in host macrophages, whereas activated macrophages are presumed to eliminate the bacteria effectively. ${ }^{22}$ Mycobacterium tuberculosis bacilli enter the macrophage via binding to several distinct cell surface molecules. Following phagocytosis, sustained intracellular bacterial growth depends on the ability to avoid destruction by macrophagemediated host defences such as lysosomal enzymes, reactive oxygen and reactive nitrogen intermediates. ${ }^{23}$

Should it occur, the killing of ingested M. tuberculosis would most likely take place within macrophage phagolysosomes. Toxic constituents found within this acidic vesicle include lysosomal hydro- lases, reactive oxygen intermediates such as $\mathrm{H}_{2} \mathrm{O}_{2}$ and $\mathrm{O}_{2}^{-}$, and reactive nitrogen intermediates such as $\mathrm{NO}$ and $\mathrm{NO}_{2}^{-}$. The resistance of several strains of $M$. tuberculosis to reactive nitrogen intermediates in vitro, generated at an acidic $\mathrm{pH}$, was found to correlate significantly with the virulence of the strain tested. ${ }^{24}$ Reactive nitrogen intermediate production by murine macrophages is an important effector mechanism against a variety of pathogens. ${ }^{25}$ In macrophages, NO and other reactive nitrogen intermediates are derived from L-arginine via an enzymatic pathway controlled by an inducible NO synthase (iNOS). ${ }^{26}$ Cytokines are powerful modulators of murine macrophage reactive nitrogen intermediate synthesis. While TNF and IFN-g are potent activators of iNOS, IL- 4 and IL-10 suppress it. $^{27-29}$

NO is known to be involved in multiple biologically important reactions, including those with transition metal ions, thiols, and redox forms of oxygen. ${ }^{30}$ Infections with pathogenic mycobacteria are associated with the development of a persistent decrease in the number of bacilli. ${ }^{31}$ This study describes an experimental model designed to test the hypothesis that this persistence may reflect in part the ability of these BCG bacilli to resist the enhanced bacteristatic and bactericidal properties acquired by host macrophages as a result of these mycobacterial infections. To examine this possibility, macrophages from mice were inoculated with viable or lysate BCG bacilli and the macrophage activation was compared. Both bacilli forms were capable of activating macrophages. Thus, the effects of lysate BCG bacilli upon macrophage activation were observed. Comparative analyses of these two types of lysate BCG in macrophages from mice demonstrated that a difference exists between these bacilli.

Resident peritoneal macrophages released a significant amount of cytokines and $\mathrm{NO}$ in response to lysate BCG bacilli. The highest levels of IFN-g were observed in macrophages from mice treated with BCG-I. The highest levels of TNF were observed for BCG-S groups and similar levels of IL- 6 were observed for both groups. Lysate BCG-I and BCG-S were compared for the ability to induce NO production in peritoneal macrophages. Cytokines participate in many physiological processes including immune and inflammatory responses. These effector molecules are produced transiently and locally control the amplitude and duration of the response. A variety of experiments showed that excessive or insufficient production may significantly contribute to the pathophysiology of a range of diseases. Particularly, cytokines released by CD4+ T cells at the onset of an immune response are thought to be decisive for pathological or physiological consequences. ${ }^{6}$ In spite of the physiological and pathological importance of TNF, the cellular factors that govern its release by macrophages are 
poorly understood, in comparison with other secretor products. ${ }^{7}$ The determination for macrophage antimycobacterial activity against $M$. bovis identified the cytokines involved in regulating the NO-mediated killing of this mycobacterium. ${ }^{32}$ Growth inhibition of $M$. bovis by IFN-g stimulated macrophage regulation by endogenous TNF and by IL-10. ${ }^{32}$

In this study, we compared the abilities of IFN-g, TNF-a to activate syngeneic murine peritoneal macrophages to inhibit the growth of intracellular BCG in vitro . IFN-g and TNF-a could activate anti-mycobacterial defence only when added to macrophage cultures after their infection with BCG.

This study also investigated the cytotoxic effects of IFN-g and TNF secreted by macrophages, and the role of NO produced by peritoneal macrophages in cytotoxic actions of cytokines. Thus, peritoneal macrophages were cultured in medium supplemented with IFN-g and TNF-a, alone or with various combinations of these cytokines. The number of viable bacilli was assessed by lysing the macrophages at $48 \mathrm{~h}$ after treatment and counting the number of cfus. Similarly, the NO production, as measured by nitrite, by macrophages, obtained from mice, in response to cytokine was assessed. There was a significant reduction in the bacilli number by both peritoneal macrophages collected after cytokine treatment. The production of NO by peritoneal macrophages was significantly increased after cytokine treatment. These results suggest that the treatment of infected macrophages with recombinant IFN-g and TNF alone markedly reduced the number of viable BCG and presented dose-dependent effects on NO production. The combination of IFN-g and TNF also reduced the number of viable bacilli and caused a greater increase in NO production. IFN-g and TNF seem to be important cytokines for the activation of mycobacterial mechanisms in murine macrophages. The activation of antibacterial effector functions in macrophages by TH1 cell-derived IFN-g is central to protection. In contrast, $\mathrm{TH} 2$ cells are only marginally involved. ${ }^{33}$ Murine peritoneal macrophages activated with IFN-g produce large quantities of NO and are efficient in killing certain intracellular pathogens. ${ }^{34}$

While IFN-g is involved in the activation of macrophage bactericidal activity, other cytokines can have antagonistic effects. IL-10, initially described as a cytokine synthesis inhibitory factor, has important regulatory effects on immune and inflammatory responses. ${ }^{35}$ The suppressive effects of IL-10 on the host response are predominantly mediated by macrophages. IL-10 inhibits the production of reactive oxygen and reactive nitrogen intermediates when macrophages are activated by IFN-g. ${ }^{36,37}$ IL-10 also inhibits TNF-a and IL-12 production by macrophages and their stimulatory effect on IFN-g production by natural killer cells. ${ }^{38,39}$
This study also showed the susceptibility of BCG bacilli by exposure to NO. The in vitro resistance of bacilli to $\mathrm{NO}$, generated at $15 \mathrm{mmol} / \mathrm{ml}$, was found to have a significant $(p<0.05)$ reduction in the number of viable BCG-I bacilli. Although increased production of NO is responsible for heightened microbicidal activity, in some cases a small number of micro-organisms can persist, leading to a subpatent infection which induce a chronic sequel $^{40,41}$ and even the complete elimination of intracellular micro-organisms. ${ }^{41}$ Various microorganisms whose development is inhibited by NO include fungi, bacteria, protozoa, helminthes and viruses. ${ }^{41}$ Recent studies with a number of microbial pathogens have established the critical role of NO and other reactive nitrogen intermediates in the microbial activity of cytokine stimulated murine macrophages. ${ }^{32,40,41}$

ACKNOWLEDGEMENTS. This work was supported by Funda ão de Amparo a Pesquisa do Estado de São Paulo (FAPESP) and Funda ão Butantan. We thank Dr Mainul Hassan and Dr Carlos Peña for critical review of the manuscript.

\section{References}

1. Kaufmann SHE. Immunity to intracellular bacteria. Annu Rev Immunol 1993; 11: 129-63.

2. Coffman RL, Mosmann TR. CD4+ T-cell subsets: regulation of differentiation and function. Res Im m unol 1991; 142: 7-79.

3. DelPrete GF, DeCarli M, Mastromauro C et al. Purified protein derivative of Mycobacterium tuberculosis and excretory-secretory antigen(s) of Toxocara canis expand in vitro human $\mathrm{T}$ cells with stable and opposite (type $1 \mathrm{~T}$ helper or type $2 \mathrm{~T}$ helper) profile of cytokine production. J Clin Invest 1991 ; 88: 346-50

4. Mosmann TR, Coffman RL. TH1 and TH2 cells: different patterns of lymphokine secretion lead to different functional properties. Annu Rev Im munol 1989; 7: 145-52.

5. Teixeira HC, Munk ME, Kaufmann SHE. Frequencies of IFN-g and IL-4 producing cells during Mycobacterium bovis BCG infection in two genetically susceptible mouse strains: role of a / $b$ T cells and NK1.1 cells. Im munol Lett 1995; 46: 15-9.

6. Van der Meide PH, Schellekeans H. Cytokines and immune response. Biotherapy 1996; 8: 243-9.

7. Stein M, Gordon S. Regulation of tumor necrosis factor (TNF) release by murine peritoneal macrophages: role of cell stimulation and specific phagocytic plasma membrane receptors. Eur J Immunol 1991; 21: 431-7.

8. Flesch IEA, Kaufmann SHE. Mycobacterial growth inhibition by interferon- $g$-activated bone marrow macrophages and differential susceptibility among strains of Mycobacterium tuberculosis. J Immunol 1987; 138: 4408-13

9. Jo T, Terada N, Takauchi Y, Nishizawa Y, Tanaka S, Kosaka N. Cytotoxic actions of cytokines on cultured mouse luteal cells are independent of nitric oxide. J Stero id Biochem Mol Biol 1995; 55: 291-6.

10. Immunological Research in Tuberculosis: Memorandum from a WHO meeting. Bull WHO 1982; 60: 723-7.

11. Petricevich VL. Compara ão dos parâmetros imunológicos induzidos experimentalmente em camundongos por duas vacinas de BCG. Doctoral Thesis. Instituto de Ciências Biomédicas da Universidade de São Paulo, 1997.

12. International Society on Toxicology. Toxicon 1992; 30: 1-12.

13. Cohn ZA, Benson B. The differentiation of mononuclear phagocytes morphology, cytochemistry and biochemistry. J Exp Med 1965; 121: 153-70.

14. Schumaker JR, O9Garra A, Schrader $\mathrm{P}$ et al. Characterization of 4 monoclonal antibodies to mouse interleukin-5 and development of mouse and human IL-5 ELISA assay. J Im munol 1988; 141: 1576-81.

15. Ruff MR, Gifford GE. Purification and physico-chemical characterization of rabbit tumor necrosis factor. J Im munol 1980; 125: 1671-77.

16. Keller R, Keist T, Wechsler A, Leist TP, van der Meide PH. Mechanisms of macrophage-mediated tumor cell killing: a comparative analysis of the roles of reactive nitrogen intermediates and tumor necrosis factor. Int $J$ Cancer 1990; 46: 682-6.

17. Silva CL, Ekizlerian SM, Fazioli RA. Role of cord factor in the modulation of infection caused by mycobacteria. Am J Pathol 1985; 118: 238-47. 
18. Lowry OH, Rosenbrough NJ, Farr AZ, Randall RJ. Protein measurement with the Folin phenol reagent. J Biol Chem 1951; 193: 265-75.

19. Douvas GS, Berger EM, Repine JE, Crowle AJ. Natural mycobacteriostatic activity in human monocyte-derived adherent cells. Am Rev Respir Dis $1986 ; 134: 44-8$.

20. Denis M. Killing of Mycobacterium tuberculosis within human monocytes: activation by cytokines and calcitriol. Clin Exp Im munol 1991; 84: 200-6.

21. Warwick-Davies J, Dhillon J, O'Brien L, Andrew PW, Lowrie DB. Apparent killing of Mycobacterium tuberculosis by cytokine-activated human monocytes can be an artifact of a cytotoxic effect on the monocytes. Clin Exp Im munol 1994; 96: 214-7.

22. Warwick-Davies J, Lowrie DB, Cole PJ. Growth hormone activation of human monocytes for superoxide production but not tumor necrosis factor production, cell adherence, or action against Mycobacterium tuberculosis. Infect Im munol 1995; 63: 4312-6.

23. Ragno S, Estrada I, Buttler R, Colston MJ. Regulation of macrophage gene expression following invasion by Mycobacterium tuberculosis. Im $m u$ nol Lett 1997; 157: 143-6.

24. O'Brien L, Carmichael J, Lowrie D, Andrew P. Strains of Mycobacterium tuberculosis differ in susceptibility to reactive nitrogen intermediates in vitro. Infect Im mun 1994; 62: 5187-90.

25. Nathan C, Hibbs J Jr. Role of nitric oxide synthesis in macrophage antimicrobial activity. Curr Opin Im munol 1991; 3: 65-70.

26. Nathan C, Xie Q-W. Nitric oxide synthesis: roles, tolls and controls. Cell 1994; 78: $915-8$.

27. Flesch IE, Kaufmann SH. Role of cytokines in tuberculosis. Im m u no bio logy 1993; 3: 316-39

28. Gazzinelli RT, Oswald IP, Hileny S, James SL, Sher A. The microbicidal activity of interferon-gamma-treated macrophages against Trypa nosome cruzi involves an L-arginine-dependent, nitrogen oxide-mediated mechanism inhabitable by interleukin-10 and transforming growth factor-beta. Eur J Im munol 1992; 10: 2501-6.

29. Oswald IP, Gazzinelli RT, Sher A, James SL. IL-10 synerziges with IL- 4 and transforming growth factor-beta to inhibit macrophage cytotoxic activity. J Im munol 1992; 148: 3578-82.

30. Stamler JS, Singel DJ, Loscalzo J. Biochemistry of nitric oxide and its redox-activated forms. Science 1992; 258: 1898-1902.

31. Orme IM, Andersen P, Boom H. T cell response to Mycobacterium tuberculosis. J Infect Dis 1993; 167: 1481-97.

32. Green SJ, Nacy CA, Schreiber RD et al. Neutralization of gamma interferon and tumor necrosis factor alpha blocks in vivo synthesis of nitrogen oxide from L-arginine and protection against Francisella tularensis infection in Mycobacterium bovis BCG-treated mice. Infect Immun 1993; 61: 689-98.

33. Kaufmann SH. Immunology of tuberculosis. Pneumologie 1995; 3: 643-8.

34. Bermudez L, Champsi J. Infection with Mycobacterium avium induces production of IL-10 (IL-10), and administration of IL-10 antibody is associated with enhanced resistance to infection in mice. Infect Im $m u n$ 1993; 61: 3093-7.

35. Moore KW, O'Garra A, de Waal Malefyt R, Vieira P, Mosmann T. Interleukin-10. Annu Rev Immunol 1993; 11: 165-90.

36. Bogdan C, VodovotzY, Nathan C. Macrophage deactivation by interleukin 10. J Exp Med 1991; 174: 1549-55.

37. Gazzinelli RT, Oswald IP, James SL, Sher A. IL-10 inhibits parasite killing and nitrogen oxide production by IFN-gamma-activated macrophage. $J$ Im munol 1992; 148: 1792-6.

38. D'Andrea A, Aste-Amezaga M, Valiente NM, Ma X, Kibin M, Trinchieri G. Interleukin 10 (IL-10) inhibits human lymphocyte interferon-g; production by suppressing natural killer cell stimulatory factor/IL-12 synthesis in accessory cells. J Exp Med 1993; 178: 1041-8.

39. Tripp CS, Wolf SF, Unanue ER. Interleukin-12 and tumor necrosis factora are costimulators of interferon- $g$ production by natural killer cells in severe combined immunodeficiency mice with listeriosis, and interleukin 10 is a physiologic antagonist. Proc Natl Acad Sci USA 1993; 90: 3725-9.

40. Drapier N. Nitric oxide and macrophages. Pathol Biol 1997; 45: $110-4$

41. Green SJ, Nacy CA. Antimicrobial and immunopathologic effects of cytokine-induced nitric oxide synthesis. Curr Opin Infect Dis 1993; 6: 383-96.

\section{Received 20 September 2000;}

accepted 16 October 2000 


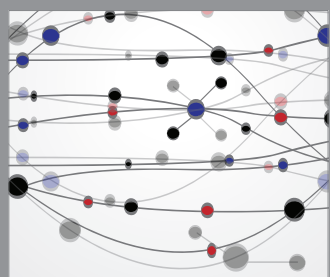

The Scientific World Journal
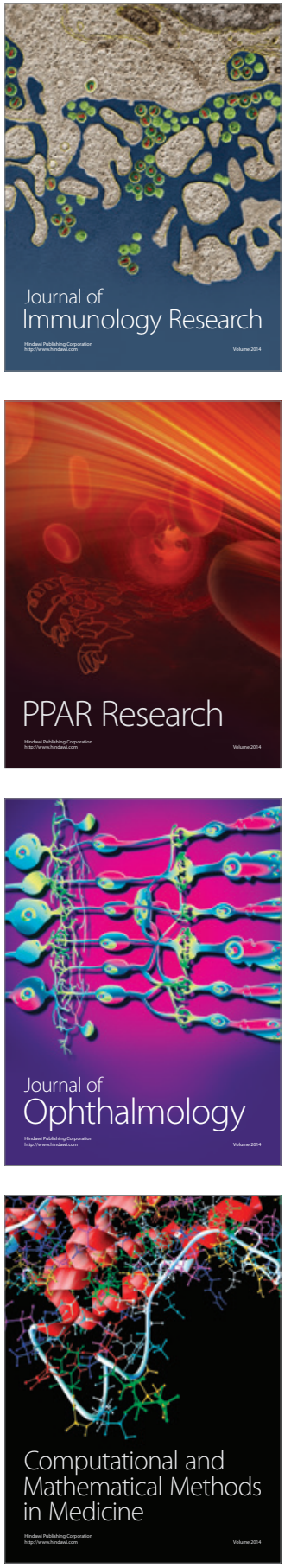

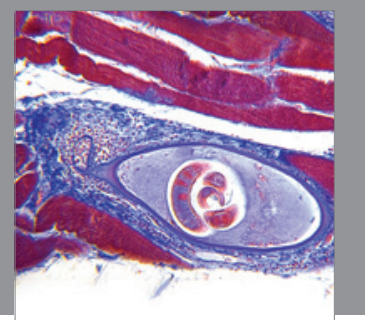

Gastroenterology

Research and Practice
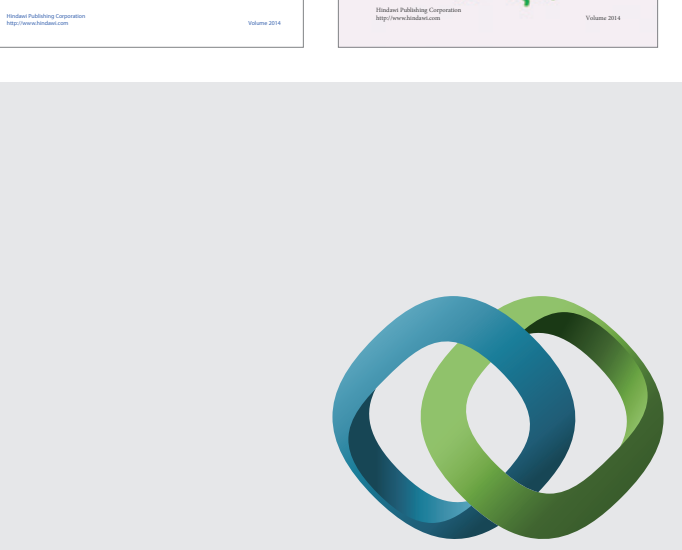

\section{Hindawi}

Submit your manuscripts at

http://www.hindawi.com
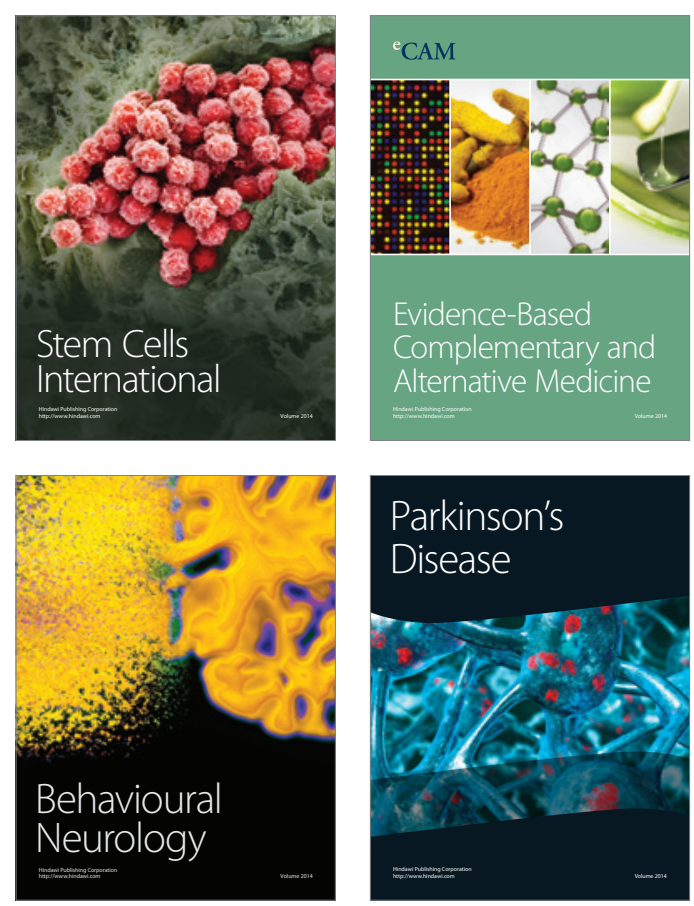

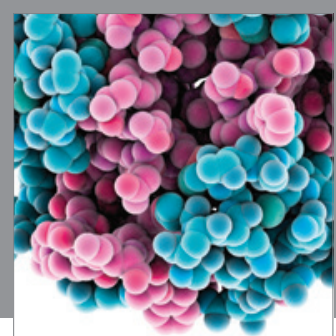

Journal of
Diabetes Research

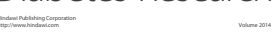

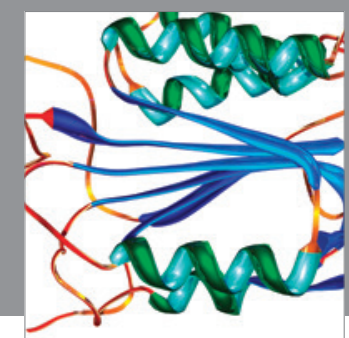

Disease Markers
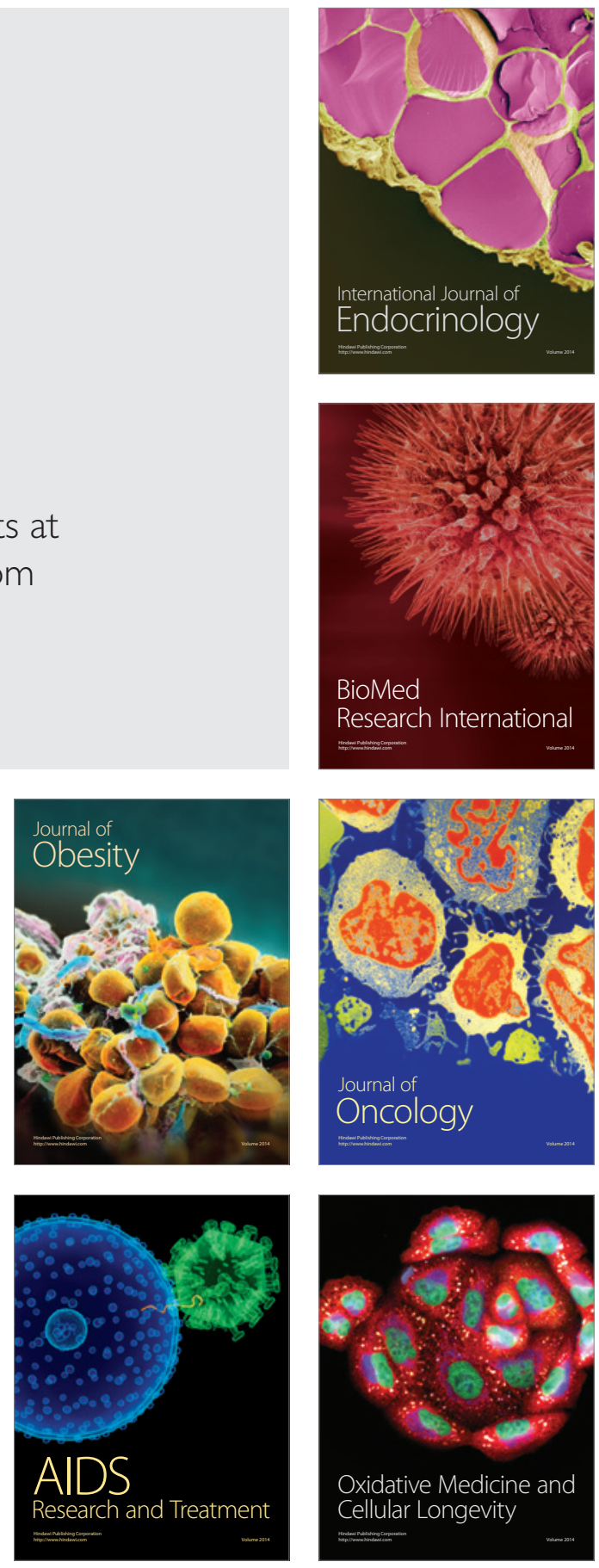